\title{
Modalities and Complications Associated With the Use of High-Flow Nasal Cannula: Experience in a Pediatric ICU
}

\author{
Florent Baudin MD MSc, Sebastien Gagnon, Benjamin Crulli MD, François Proulx MD, \\ Philippe Jouvet MD PhD, and Guillaume Emeriaud MD PhD
}

\begin{abstract}
BACKGROUND: High-flow nasal cannula (HFNC) therapy is increasingly used in pediatric ICUs as an intermediate level of support between conventional oxygen delivery and noninvasive ventilation (NIV). The safety of HFNC has seldom been studied, and some cases of barotrauma have been reported. This retrospective study aims to describe HFNC use in a tertiary care pediatric ICU, with a focus on the complications associated with this therapy. METHODS: Between January 2013 and January 2014, all children $<18$ y old treated with HFNC in the pediatric ICU were included. Demographic data, HFNC settings, chest radiograph reports, and blood gas values were gathered from the electronic medical records. Episodes of pneumothorax, pneumomediastinum, and significant epistaxis were noted. Pneumothorax was distinguished from chest tube-related air leak (frequent after cardiac surgery), which was defined as a small pneumothorax with no clinical impact that resolved spontaneously after chest tube removal. RESULTS: During the 1-y study period, there were 177 HFNC episodes, involving 145 subjects with a median (interquartile range) age of 8 (2-28) months. HFNC was used as primary support in $31 \%$ of episodes, after extubation in $36 \%$ and after NIV in $18 \%$. HFNC was administered exclusively for nitric oxide delivery in $16 \%$ of episodes. Two children $(1 \%)$ developed new pneumothoraces that required chest tube insertion, whereas $5(3 \%)$ chest tube-related air leaks were noted. One $(0.6 \%)$ episode of significant epistaxis was noted. Among 6 preexisting pneumothoraces, none worsened under HFNC. Failure of HFNC occurred in 32 episodes, requiring transition to NIV in 28 cases and endotracheal intubation in 5 cases. CONCLUSIONS: Support with HFNC following a clinical protocol in pediatric ICUs was associated with a relatively low rate of complications. Since HFNC use is increasing, further evidence is needed to confirm its efficacy and safety. Key words: high-flow nasal cannula; children; barotrauma; pediatric ICU; pneumothorax; noninvasive ventilation; complications. [Respir Care 2016;61(10):1305-1310. (C) 2016 Daedalus Enterprises]
\end{abstract}

\section{Introduction}

The high-flow nasal cannula (HFNC) is a recent device used in ICUs that can provide an intermediate level of

\footnotetext{
All authors are affiliated with the Department of Pediatrics, CHU SainteJustine, Université de Montréal, Montreal, Quebec, Canada. Dr Baudin is also affiliated with the Pediatric Intensive Care Unit, Hôpital FemmeMère-Enfant, Hospices Civils de Lyon, Bron, France.
}

Supplementary material related to this paper is available at http:// www.rcjournal.com.

Mr Gagnon was supported by the COPSE program of the Université de Montréal. Dr Emeriaud holds a Clinical Research Scholarship from the support between conventional low-flow oxygen delivery and noninvasive ventilation (NIV). HFNC delivers humid-

\footnotetext{
Fonds de la Recherche en Santé du Québec. The authors have disclosed no conflicts of interest.

The first 2 authors contributed equally to this work.

Dr Baudin presented a version of this paper at the 43rd International Congress on Intensive Care Medicine of the Société de Réanimation de Langue Française (SRLF), held January 21-23, 2015, in Paris, France.

Correspondence: Guillaume Emeriaud MD PhD, Department of Pediatrics, CHU Sainte Justine, 3175 Chemin Côte Sainte Catherine, Montreal, Quebec H3T 1C5, Canada. E-mail: guillaume.emeriaud@umontreal.ca.
}

DOI: $10.4187 /$ respcare. 04452 


\section{Modalities and Complications Associated With Use of HFNC}

ified and heated gas at a high flow that can match or exceed the patient's inspiratory flow. ${ }^{1}$ Washout of the nasopharyngeal space limits $\mathrm{CO}_{2}$ re-inhalation and reduces anatomical dead space by creating a pharyngeal reserve of fresh gas for subsequent inspiration..$^{2-4}$ During the inspiratory phase, HFNC maintains a pharyngeal pressure 5 and therefore reduces inspiratory nasopharyngeal resistance. In addition, a certain level of PEEP may prevent small airway collapse during expiration. This pressure is variable (between 2 and $7 \mathrm{~cm} \mathrm{H}_{2} \mathrm{O}$ in children) and depends on flow, mouth opening, and cannula size. ${ }^{6-10}$ These different mechanisms result in improved oxygenation ${ }^{11}$ and reduced work of breathing, depending on flow. ${ }^{5,12,13}$ HFNC use is simple and well tolerated by patients. ${ }^{14,15}$ This may contribute to its growing popularity in neonatal and pediatric ICUs. ${ }^{16,17}$

Following widespread implementation in pediatric ICUs, the safety of HFNC has been questioned..$^{717-19}$ In particular, Hegde and Prodhan ${ }^{19}$ reported 3 cases of severe barotrauma ( 2 pneumothoraces and one pneumomediastinum) possibly related to HFNC. A meta-analysis found no evidence available to determine the safety of HFNC in children, and the authors concluded that further studies were needed in that field. ${ }^{20}$

In 2012, HFNC technology was introduced in our pediatric ICU along with a clinical protocol aiming to limit the flow administered. We reviewed our experience with HFNC therapy to describe the modalities and report the complications associated with this therapy in our pediatric ICU.

\section{Methods}

We conducted a retrospective observational study including all subjects $<18$ y old consecutively admitted to the pediatric ICU of university-affiliated Sainte-Justine Hospital (Montreal, Canada) and treated with high-flow (>5 L/min) nasal cannula between January 8, 2013 and January 7, 2014. The study protocol, including waiver of consent, was approved by the institutional ethics committee (approval number 3944).

\section{Data Collection}

By querying the database from the electronic medical records software (IntelliSpace Critical Care and Anesthesia [ICCA], Philips Healthcare, Toronto, Canada), we identified all subjects treated with HFNC. HFNC episodes separated by at least $48 \mathrm{~h}$ were considered as distinct. Clinical, laboratory, and epidemiological data were extracted. Severity of illness was estimated using the Pediatric Risk of Mortality II score ${ }^{21}$ at admission and the Pediatric Logistic Organ Dysfunction score ${ }^{22}$ on the day of HFNC initiation. Indication, duration, and settings of HFNC use were recorded. Chest radiograph reports from the day preceding initiation of HFNC therapy to the day following its termination were col-

\section{QUICK LOOK}

\section{Current knowledge}

High-flow nasal cannula (HFNC) therapy is increasingly used in critically ill children as an intermediate level of support between conventional oxygen delivery and noninvasive ventilation, although supportive evidence is lacking. In particular, the safety of HFNC and the risk of barotrauma have seldom been studied.

\section{What this paper contributes to our knowledge}

We systematically reviewed the use of HFNC and investigated the occurrence of complications in all consecutive children treated with this technology in a pediatric ICU during a 1-y period. New pneumothoraces or significant epistaxis each occurred in $1 \%$ of cases. Support with HFNC following a clinical protocol in a pediatric ICU seems to be associated with relatively rare but potentially serious complications.

lected. Chest radiographs were not performed systematically but at the discretion of the physician.

\section{Respiratory Support}

During the study period, the Optiflow RT330 (Fisher \& Peykel Healthcare, Auckland, New Zealand) circuit was used. HFNC use was guided by a standardized clinical protocol (see the supplementary materials at http:// www.rcjournal.com). Cannula size was selected using the subject's weight and nasal anatomy. Flow was initially set at $1 \mathrm{~L} / \mathrm{kg} / \mathrm{min}$ (up to $15 \mathrm{~L} / \mathrm{min}$ ) and progressively increased according to the subject's response and tolerance, up to a maximal rate determined with the patient's weight and prong size.

\section{Outcomes}

The primary outcome was the development of major complications using HFNC, including: pneumothorax, pneumomediastinum, or clinically important epistaxis. Episodes of barotrauma were recorded using radiology reports of all chest radiographs performed between the day before HFNC therapy and the day after its cessation. Small pneumothoraces are frequently observed in the postoperative period after cardiac surgery due to the manipulation of chest tubes. Those small pneumothoraces, which had no clinical impact and resolved rapidly after chest tube removal, were therefore defined as chest tube-related air leaks. Epistaxis was considered as clinically important when 


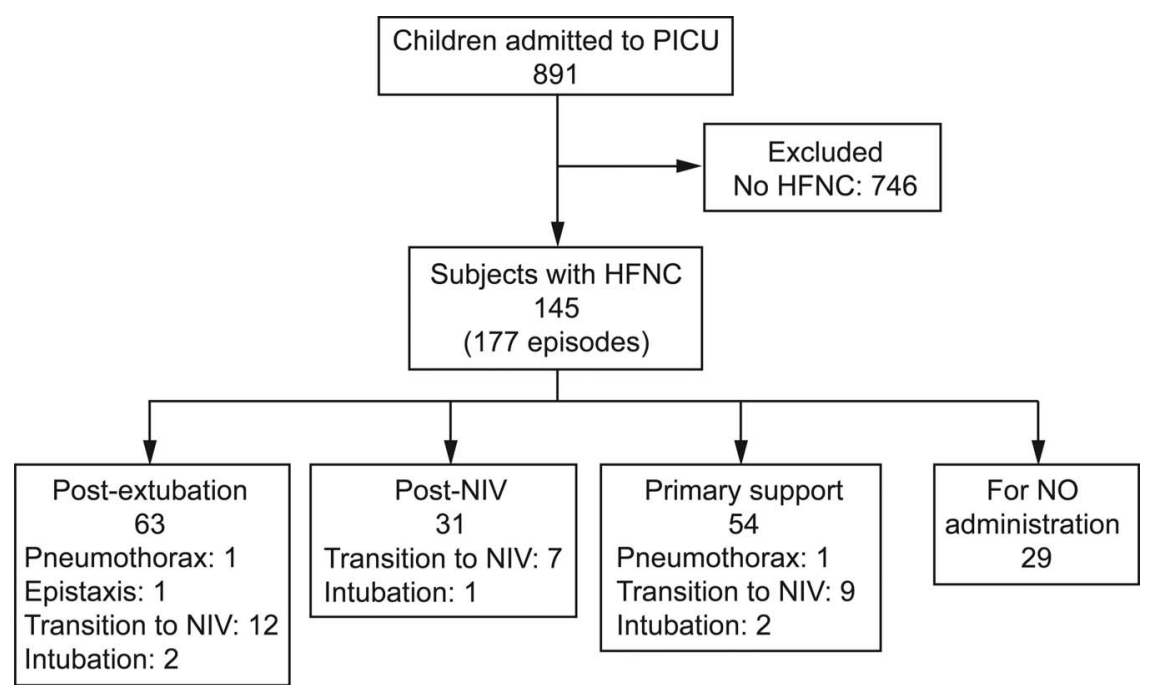

Fig. 1. Flow chart. PICU = pediatric ICU, HFNC = high-flow nasal cannula, NIV = noninvasive ventilation, NO = nitric oxide.

an otolaryngologist consultation was required to discuss packing or other local treatment.

Tolerance of HFNC was assessed using the evolution of clinical (at initiation and after 2 and $24 \mathrm{~h}$ of treatment) and biochemical parameters. The latter included blood gas values (capillary or arterial) at 3 separate times: baseline (within $4 \mathrm{~h}$ before HFNC initiation), early (first value between $30 \mathrm{~min}$ and $6 \mathrm{~h}$ after HFNC initiation), and late (first value between 12 and $24 \mathrm{~h}$ after HFNC initiation). HFNC failure was defined as the requirement of noninvasive or invasive ventilation within $24 \mathrm{~h}$ of HFNC termination. When HFNC was used exclusively for nitric oxide administration, complications were recorded, but failure and clinical tolerance were not assessed. The number of chest radiographs and blood gas analyses performed during HFNC therapy was recorded.

\section{Statistical Analysis}

Statistical analysis was performed using SPSS 20 (IBM Corporation, Armonk, New York). Qualitative variables are reported as numbers and percentages, whereas quantitative variables are reported as mean $\pm \mathrm{SD}$ or as median (interquartile range $[\mathrm{IQR}]$ ) when appropriate. The rate of complications is reported per HFNC episode and per 100 HFNC days, with a 95\% CI. The chi-square test was used to compare qualitative variables between subgroups. The Friedman test was used to compare clinical parameters and blood gas values at different times. Post hoc analysis was conducted using Wilcoxon signed-rank tests, with a Bonferroni correction for multiple comparisons. Differences were considered statistically significant at $P<.01$.

\section{Results}

\section{Subjects}

During the 1-y study period, 891 children were admitted to the pediatric ICU, and 145 (16\%) were exposed to HFNC (70 boys and 75 girls) over a total of 177 episodes (Fig. 1). The median (IQR) age was 8 (2-28) months, and weight was $6.5(4-12) \mathrm{kg}$. Congenital heart disease accounted for $52 \%$ of the study population, including 59 subjects $(41 \%)$ hospitalized after cardiac surgery. Twentythree children $(16 \%)$ were admitted for bronchiolitis, and $10(7 \%)$ were admitted for pneumonia. Chronic respiratory disease was present in 20 subjects (14\%). At admission, the Pediatric Risk of Mortality II score was 10 (6-15), whereas the Pediatric Logistic Organ Dysfunction score at initiation of HFNC therapy was 10 (1-11). Indication, parameters, and duration of HFNC episodes are reported in Table 1 . In 16\% of cases, no ventilatory support was needed, and HFNC was used exclusively for nitric oxide delivery. The median number (IQR) of blood gas analyses and chest radiographs performed per episode was 4 (2-7) and 1 (1-3), respectively.

\section{Complications}

Six preexisting pneumothoraces $(3 \%)$ were identified before HFNC initiation, none of which worsened under HFNC. Two episodes (1\%) of new pneumothoraces and 5 episodes $(3 \%)$ of chest tube-related air leaks were noted after the start of HFNC (see Table 1). One episode of clinically important epistaxis $(0.6 \%)$ occurred during HFNC therapy. The rate of complications was $0.9 / 100$ HFNC treatment days (95\% CI 0-1.9 / 100 HFNC days). 
Table 1. Characteristics and Outcome of High-Flow Nasal Cannula Episodes

\begin{tabular}{|c|c|}
\hline Characteristics & Values \\
\hline Preexisting pneumothorax, $n(\%)$ & $6(3)$ \\
\hline \multicolumn{2}{|l|}{ HFNC indication, $n(\%)$} \\
\hline Post-extubation & $63(36)$ \\
\hline Post-NIV & $31(18)$ \\
\hline Primary support & $54(31)$ \\
\hline NO administration & $29(16)$ \\
\hline Duration of HFNC episode, median (IQR) h & $36(17-61)$ \\
\hline \multicolumn{2}{|l|}{ Initial HFNC parameters, median (IQR) } \\
\hline $\mathrm{F}_{\mathrm{IO}_{2}}(\%)$ & $35(30-50)$ \\
\hline Flow $(\mathrm{L} / \mathrm{kg} / \mathrm{min})$ & $1.25(0.89-1.76)$ \\
\hline \multicolumn{2}{|l|}{ Maximum HFNC parameters, median (IQR) } \\
\hline $\mathrm{F}_{\mathrm{IO}_{2}}, \%$ & $40(30-60)$ \\
\hline Flow $(\mathrm{L} / \mathrm{kg} / \mathrm{min})$ & $1.36(0.98-1.86)$ \\
\hline Complications, $n(\%)$ & $8(5)$ \\
\hline New pneumothorax & $2(1)$ \\
\hline New pneumomediastinum & 0 \\
\hline Chest tube-related air leak & $5(3)$ \\
\hline Clinically significant epistaxis & $1(1)$ \\
\hline HFNC failure, $n(\%)$ & $32(22)^{*}$ \\
\hline Tracheal intubation & $5(3)^{*}$ \\
\hline Transition to NIV & $28(19)^{*}$ \\
\hline \multicolumn{2}{|l|}{ Monitoring per episode, median (IQR) } \\
\hline Chest radiograph & $1(1-3)$ \\
\hline Blood gas analyses & $4(2-7)$ \\
\hline \multicolumn{2}{|c|}{$\begin{array}{l}n=177 . \\
\text { * Episodes of high-flow nasal cannula use for nitric oxide administration exclusively }(n=29) \\
\text { were excluded from these ratios. } \\
\text { HFNC = high-flow nasal cannula } \\
\text { NIV = noninvasive ventilation } \\
\text { NO = nitric oxide } \\
\text { IQR = interquartile range }\end{array}$} \\
\hline
\end{tabular}

One pneumothorax occurred in a 2-y-old child with a history of bronchopulmonary dysplasia and asthma admitted for severe bronchospasm. HFNC therapy was started immediately upon pediatric ICU admission as primary support. Although no sign of pneumothorax was present at admission, a small right-sided pneumothorax was noted after $24 \mathrm{~h}$ of HFNC therapy; it had progressed to both sides on the following day. Bilateral chest tubes were inserted. HFNC was weaned after $51 \mathrm{~h}$ without need for subsequent NIV or endotracheal intubation.

The second pneumothorax occurred in a 13-y-old girl admitted after a heart transplant. HFNC was initiated after extubation on postoperative day 1 mainly to administer inhaled nitric oxide. The next day, the chest radiograph showed a right-sided pneumothorax, which required drainage for $3 \mathrm{~d}$.

\section{Protocol Deviation}

In 3 subjects, the flow exceeded the maximum suggested by our protocol. In two infants (weighing 3.5 and
$4.1 \mathrm{~kg}$ ), the flow was temporarily increased to 12 and 15 $\mathrm{L} / \mathrm{min}$, respectively. A child weighing $7 \mathrm{~kg}$ was treated with a flow of $20 \mathrm{~L} / \mathrm{min}$. These 3 children were later switched to NIV due to worsening respiratory failure, and none developed complications.

\section{Tolerance and Failure}

HFNC failure occurred in 32 cases (22\%), requiring transition to NIV in 28 subjects and endotracheal intubation in 5 cases (see Table 1). One subject transitioned to NIV was subsequently intubated. Evolution of clinical and biochemical parameters ( $\mathrm{pH}$ and $\mathrm{P}_{\mathrm{CO}_{2}}$ ) after HFNC initiation is presented in Table 2 . Early and late blood gas values were obtained 1.2 (0.9-2.7) h and 17 (14-19) h after HFNC initiation, respectively. A slight but significant improvement of $\mathrm{pH}$ and $\mathrm{P}_{\mathrm{CO}_{2}}$ was observed after HFNC initiation. With regard to clinical parameters, there was a significant decrease in heart rate and mean blood pressure, whereas breathing frequency and $\mathrm{S}_{\mathrm{pO}_{2}}$ remained stable (see Table 2).

\section{Discussion}

In this retrospective study, we report that major complications of HFNC were relatively rare $(0.9$ per 100 HFNC treatment days), despite an increasing number of subjects being treated with HFNC in our pediatric ICU. HFNC has become an interesting alternative for delivery of noninvasive respiratory support in neonates and children because it is simple to use and well tolerated. However, there is little evidence in the literature regarding the safety of HFNC use in a pediatric ICU.

Over $1 \mathrm{y}$, we observed a low rate of barotrauma (2 episodes, 1\%) and of clinically important epistaxis (1 episode, $0.6 \%$ ). The role of HFNC in the development of these pneumothoraces is difficult to ascertain. In the first subject, severe bronchospasm alone may have led to a pneumothorax through hyperinflation. In the second, air leak may have been secondary to the surgery rather than the ventilator support. Interestingly, among the 6 preexisting pneumothoraces, none worsened under HFNC. This incidence of preexisting pneumothoraces is explained by the high proportion of postoperative patients in our population, and our data suggest that HFNC may still be used, with caution, in these patients. HFNC may be considered as positive pressure support and not merely as a device for oxygen delivery. Physiological studies have confirmed that HFNC can generate positive pressure depending on flow. $3,5,7$ In our study, the flow was limited by a protocol. This limit was exceeded in only 3 subjects, who subsequently required NIV. The standardized protocol may therefore have contributed to preventing barotrauma. The role of a surveillance bias appears unlikely, since subjects were closely monitored with a median of 1 chest radiograph and 4 blood gas analyses being performed during HFNC episodes. 


\section{Modalities and Complications Associated With Use of HFNC}

Table 2. Evolution of Clinical Parameters and Blood Gas After Initiation of High-Flow Nasal Cannula in the Subgroup of Subjects Treated With High-Flow Nasal Cannula for Reasons Other Than Nitric Oxide Delivery

\begin{tabular}{|c|c|c|c|c|}
\hline Parameters & $\begin{array}{c}\text { Baseline } \\
\text { (Before HFNC) }\end{array}$ & $\begin{array}{l}\text { Early HFNC Period } \\
\quad(0.5-6 \mathrm{~h})^{*}\end{array}$ & $\begin{array}{l}\text { Late HFNC Period } \\
\qquad(12-24 \mathrm{~h}) \dagger\end{array}$ & $P \ddagger$ \\
\hline Clinical parameters & $n=147$ & $n=146$ & $n=92$ & \\
\hline Heart rate, median (IQR) beats/min & $148(132-163)$ & $142(124-155)$ & $140(124-152)$ & $<.001 \S$ \\
\hline Mean arterial pressure, median (IQR) $\mathrm{mm} \mathrm{Hg}$ & $71(61-84)$ & $68(58-77)$ & $65(55-79)$ & $<.001 \S$ \\
\hline Breathing frequency, median (IQR) breaths/min & $38(27-52)$ & $40(29-53)$ & $43(31-56)$ & .61 \\
\hline $\mathrm{S}_{\mathrm{PO}_{2}}$, median (IQR) \% & $99(94-100)$ & $98(95-100)$ & $98(94-100)$ & .49 \\
\hline $\begin{array}{l}\text { Capillary or arterial blood gas } \\
\mathrm{pH} \text { median (IOR) }\end{array}$ & $\begin{array}{c}n=80 \\
7.35(7.31-7.41)\end{array}$ & $\begin{array}{c}n=104 \\
7.37(7.33-7.42)\end{array}$ & $\begin{array}{c}n=86 \\
7.39(7.35-7.42)\end{array}$ & 0078 \\
\hline $\mathrm{P}_{\mathrm{CO}_{2}}$, median (IQR) $\mathrm{mm} \mathrm{Hg}$ & $46(41-54)$ & $45(40-52)$ & $44(40-50)$ & $.003 \S$ \\
\hline \multicolumn{5}{|c|}{$\begin{array}{l}\text { * Early HFNC period data correspond to the first values observed between } 30 \mathrm{~min} \text { and } 6 \mathrm{~h} \text { after HFNC initiation. } \\
\dagger \text { Late HFNC period data correspond to the first values observed between } 12 \text { and } 24 \mathrm{~h} \text { after HFNC initiation. } \\
\text { † Friedman test; post hoc significant difference at } P<.01 \text {. } \\
\S \text { Significant difference between baseline and early HFNC period and between baseline and late HFNC period. } \\
\text { HFNC = high-flow nasal cannula } \\
\text { IQR = interquartile range }\end{array}$} \\
\hline
\end{tabular}

The safety of HFNC in critically ill children has seldom been studied. A meta-analysis evaluated the safety and efficacy of this technology, but no studies of sufficient quality could be identified. The authors concluded that there was a need for further work to determine which subgroups could benefit from HFNC therapy. ${ }^{20}$ Schlapbach et $\mathrm{al}^{23}$ reported their experience with HFNC (with flow set at $2 \mathrm{~L} / \mathrm{kg} / \mathrm{min}$ ) during pediatric interhospital transport. No pneumothorax and no death occurred during the transport of 150 children. Schibler et al ${ }^{17}$ conducted a retrospective study in 248 children, where they reported no pneumothorax or mucosal injuries. The flow in this study was set at $8 \mathrm{~L} / \mathrm{min}$ in children weighing $5.5(4.0-7.8) \mathrm{kg}$.

In our study, we observed a failure rate of $22 \%$, with intubation being required in 5 subjects (3\%). This rate of HFNC failure is similar to those in previous reports, but we observed a relatively low intubation rate. In the study by Schibler et al, ${ }^{17} 12 \%$ of children required invasive ventilation; this rate was lower in the bronchiolitis subgroup (4\%). McKiernan et $\mathrm{al}^{24}$ reported an intubation rate of $9 \%$ in children with bronchiolitis. The low rate in our study is probably explained in part by the high prevalence of subjects with bronchiolitis and also because the indication for HFNC was respiratory failure in only $36 \%$ of cases. We may also hypothesize that HFNC was frequently used preventively or in subjects with moderate signs of respiratory failure. As observed with other noninvasive devices, $\mathrm{pH}$, $\mathrm{P}_{\mathrm{CO}_{2}}$, and heart rate quickly improved after HFNC initiation. ${ }^{25}$ These parameters would, however, possibly have improved spontaneously over time even without HFNC.

As with NIV, the risk of delaying intubation in patients with poor cardiorespiratory reserve must be considered. Intubation after failure of conventional NIV is associated with a particularly high risk of adverse events. ${ }^{26}$ In adults, an increased risk of mortality has been reported in subjects being treated with HFNC therapy during prolonged periods of time before endotracheal intubation. ${ }^{27}$ The number of subjects requiring intubation in our study was too low to look for an association between HFNC duration and failure. HFNC use could also result in the multiplication of support levels, which could lead to further delay in establishing adequate ventilation and increased costs. This did not seem to be the case in our study because only one subject was intubated after an additional trial of NIV following HFNC failure.

Our study has several limitations. Although retrospective in design, an objective and systematic strategy was used to query the electronic medical record database to obtain exhaustive data. The definition of epistaxis may appear particularly restrictive, and the episodes of lesser severity were not identified. This was intentional because we aimed to focus on significant complications. A large proportion of the study participants were either infants or patients hospitalized after surgery. It would be important in future studies to specifically assess complications associated with HFNC in older children and in non-surgical patients. The study sample size and the low rate of observed complications did not allow for stratification of the results according to subject age, disease severity, HFNC flow, or HFNC indication. Finally, this study was conducted in a single center with a specific protocol guiding HFNC use, thereby limiting its external validity.

\section{Conclusions}

Our practice of using HFNC in critically ill children using limited flow as guided by a clinical protocol was associated with a low incidence of complications. Further 


\section{Modalities and Complications Associated With Use of HFNC}

prospective studies are needed to confirm the efficacy of HFNC therapy and to evaluate the risks associated with HFNC use in different settings.

\section{REFERENCES}

1. Sim MAB, Dean P, Kinsella J, Black R, Carter R, Hughes M. Performance of oxygen delivery devices when the breathing pattern of respiratory failure is simulated. Anaesthesia 2008;63(9):938-940.

2. Delisle S, Ouellet P. Principes de physiologie et d'anatomie fonctionnelle de la thérapie par haut débit humidifié (THDH). Réanimation 2012;21(1):20-25.

3. Dysart K, Miller TL, Wolfson MR, Shaffer TH. Research in high flow therapy: mechanisms of action. Respir Med 2009;103(10):14001405.

4. Milési C, Boubal M, Jacquot A, Baleine J, Durand S, Odena MP, et al. High-flow nasal cannula: recommendations for daily practice in pediatrics. Ann Intensive Care 2014;4:29.

5. Milési C, Baleine J, Matecki S, Durand S, Combes C, Novais ARB, et al. Is treatment with a high flow nasal cannula effective in acute viral bronchiolitis? A physiologic study. Intensive Care Med 2013; 39(6):1088-1094.

6. Volsko TA, Fedor K, Amadei J, Chatburn RL. High flow through a nasal cannula and CPAP effect in a simulated infant model. Respir Care 2011;56(12):1893-1900.

7. Spentzas T, Minarik M, Patters AB, Vinson B, Stidham G. Children with respiratory distress treated with high-flow nasal cannula. J Intensive Care Med 2009;24(5):323-328.

8. Hasan RA, Habib RH. Effects of flow rate and airleak at the nares and mouth opening on positive distending pressure delivery using commercially available high-flow nasal cannula systems: a lung model study. Pediatr Crit Care Med 2011;12(1):e29-e33.

9. Kubicka ZJ, Limauro J, Darnall RA. Heated, humidified high-flow nasal cannula therapy: yet another way to deliver continuous positive airway pressure? Pediatrics 2008;121(1):82-88.

10. Parke RL, Eccleston ML, McGuinness SP. The effects of flow on airway pressure during nasal high-flow oxygen therapy. Respir Care 2011;56(8):1151-1155.

11. Frizzola M, Miller TL, Rodriguez ME, Zhu Y, Rojas J, Hesek A, et al. High-flow nasal cannula: impact on oxygenation and ventilation in an acute lung injury model. Pediatr Pulmonol 2011;46(1):67-74.

12. Pham TMT, O'Malley L, Mayfield S, Martin S, Schibler A. The effect of high flow nasal cannula therapy on the work of breathing in infants with bronchiolitis. Pediatr Pulmonol 2015;50(7):713-720.
13. Rubin S, Ghuman A, Deakers T, Khemani R, Ross P, Newth CJ. Effort of breathing in children receiving high-flow nasal cannula. Pediatr Crit Care Med 2014;15(1):1-6.

14. Benzidi Y, Robriquet L. Oxygen therapy in all its forms: how to administer oxygen in 2014? Réanimation 2014;23(1):65-75.

15. Ward JJ. High-flow oxygen administration by nasal cannula for adult and perinatal patients. Respir Care 2013;58(1):98-122.

16. Lee JH, Rehder KJ, Williford L, Cheifetz IM, Turner DA. Use of high flow nasal cannula in critically ill infants, children, and adults: a critical review of the literature. Intensive Care Med 2013;39(2): 247-257.

17. Schibler A, Pham TMT, Dunster KR, Foster K, Barlow A, Gibbons $\mathrm{K}$, Hough JL. Reduced intubation rates for infants after introduction of high-flow nasal prong oxygen delivery. Intensive Care Med 2011; 37(5):847-852.

18. Sivieri EM, Gerdes JS, Abbasi S. Effect of HFNC flow rate, cannula size, and nares diameter on generated airway pressures: an in vitro study. Pediatr Pulmonol 2013;48(5):506-514.

19. Hegde S, Prodhan P. Serious air leak syndrome complicating highflow nasal cannula therapy: a report of 3 cases. Pediatrics 2013; 131(3):e939-e944.

20. Mayfield S, Jauncey-Cooke J, Hough JL, Schibler A, Gibbons K, Bogossian F. High-flow nasal cannula therapy for respiratory support in children. Cochrane Database Syst Rev 2014;(3):CD009850.

21. Pollack MM, Ruttimann UE, Getson PR. Pediatric risk of mortality (PRISM) score. Crit Care Med 1988;16(11):1110-1116.

22. Leteurtre S, Martinot A, Duhamel A, Proulx F, Grandbastien B, Cotting J, et al. Validation of the paediatric logistic organ dysfunction (PELOD) score: prospective, observational, multicentre study. Lancet 2003;362(9379):192-197.

23. Schlapbach LJ, Schaefer J, Brady AM, Mayfield S, Schibler A. High-flow nasal cannula (HFNC) support in interhospital transport of critically ill children. Intensive Care Med 2014;40(4):592-599.

24. McKiernan C, Chua LC, Visintainer PF, Allen H. High flow nasal cannulae therapy in infants with bronchiolitis. J Pediatr 2010;156(4): 634-638.

25. Essouri S, Chevret L, Durand P, Haas V, Fauroux B, Devictor D. Noninvasive positive pressure ventilation: five years of experience in a pediatric intensive care unit. Pediatr Crit Care Med 2006;7(4):329334.

26. Crulli B, Loron G, Nishisaki A, Harrington K, Essouri S, Emeriaud G. Safety of paediatric tracheal intubation after non-invasive ventilation failure. Pediatr Pulmonol 2016;51(2):165-172.

27. Kang BJ, Koh Y, Lim CM, Huh JW, Baek S, Han M, et al. Failure of high-flow nasal cannula therapy may delay intubation and increase mortality. Intensive Care Med 2015;41(4):623-632.

This article is approved for Continuing Respiratory Care Education credit. For information and to obtain your CRCE

(free to AARC members) visit www.rcjournal.com

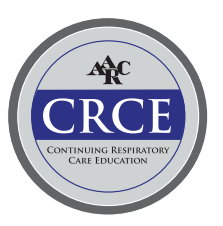

